\title{
Success Rate of Airway Devices Insertion: Laryngeal Mask Airway Versus Supraglottic Gel Device
}

\author{
Alireza Pournajafian ${ }^{1,}$; Mahzad Alimian ${ }^{2}$; Faranak Rokhtabnak ${ }^{1}$; Mohammadreza \\ Ghodraty ${ }^{1}$; Mozhgan Mojri ${ }^{1}$
}

${ }_{1}^{1}$ Department of Anesthesiology and Pain Medicine, Firoozgar Hospital, Iran University of Medical Sciences, Tehran, Iran

${ }^{2}$ Department of Anesthesiology, Rasool-e-Akram Hospital, Iran University of Medical Sciences, Tehran, Iran

${ }^{*}$ Corresponding author: Alireza Pournajafian, Department of Anesthesiology and Pain Medicine, Firoozgar Hospital, Iran University of Medical Sciences, Tehran, Iran. Tel: +982182141360, Fax:+98-2188942622, E-mail: pournajafian.ar@iums.ac.ir

Received: July 15, 2014; Revised: August 5, 2014; Accepted: August 11, 2014

Background:The main important method for airway management during anesthesia is endotracheal intubation. Laryngeal mask airway (LMA) and supraglottic gel device (I-Gel) are considered alternatives to endotracheal tube.

Objectives: This study sought to assess the success rate of airway management using LMA and I-Gel in elective orthopedic surgery.

Patients and Methods: This single-blinded randomized clinical trial was performed on 61 ASA Class 1 and 2 patients requiring minor orthopedic surgeries. Patients were randomly allocated to two groups of LMA and I-Gel. Supraglottic airway placement was categorized into three groups regarding the number of placement attempts, i.e. on the first, second, and third attempts. Unsuccessful placement on the third attempt was considered failure and endotracheal tube was used in such cases. The success rate, insertion time, and postoperative complications such as bleeding, sore throat, and hoarseness were recorded.

Results: In the I-Gel group, the success rate was $66.7 \%$ for placement on the first attempt, $16.7 \%$ for the second, and $3.33 \%$ for the third attempt. In the LMA group, the success rates were $80.6 \%$ and $12.9 \%$ for the first and second attempts, respectively. Failure in placement occurred in four cases in the I-Gel and two cases in LMA groups. The mean insertion time was not significantly different between two groups (21.35 seconds in LMA versus 27.96 seconds in $\mathrm{I}-\mathrm{Gel}, \mathrm{P}=\mathbf{0 . 2}$ ). The incidence of postoperative complications was not significantly different between study groups.

Conclusions: I-Gel can be inserted as fast as LMA with adequate ventilation in patients and has no major airway complications. Therefore, it could be a good alternative to LMA in emergency airway management or general anesthesia.

Keywords: Laryngeal Mask; Mechanical Ventilation; General Anesthesia

\section{Background}

Proper airway management is the most important task for an anesthesiologist during general anesthesia. Endotracheal intubation is the gold standard for airway management; however, this maneuver requires expertise and lots of clinical experience $(1,2)$. Laryngoscopy and endotracheal intubation trigger the sympathetic reflex response, increase the plasma level of catecholamines, and cause hypertension, tachycardia, myocardial infarction, decreased myocardial contractility, and ventricular arrhythmia, all of which can be life-threatening $(3,4)$. Attempts to prevent complications related to endotracheal intubation and laryngoscopy led to the introduction of new airway devices, namely, laryngeal mask airway (LMA) and I-Gel. LMA has been successfully used for more than a decade. It has an airway tube that connects to a mask with a cuff that is inflated. It has been used in managing difficult airways and in different kinds of surgeries with minimal changes in hemodynamic responses (5-7). Incorrect positioning of the LMA leads to aspiration, pneumonia, air leakage, and partial airway obstruction with an overinflated or malpositioned cuff, and might even trigger the sympathetic reflex responses (8-12). I-Gel is a supraglottic airway device, renowned for its easy application and simplicity, particularly for the resuscitation procedures (13). I-Gel works in accordance with human anatomy with lesser trauma to the pharynx, larynx, and supraglottic areas due to its minimal displacement. This device is capable of separating the gastrointestinal and the respiratory systems and possesses a tube to suction the stomach contents. Its rapid placement, possession of a noninflatable cuff, and disposability are among the other advantages of this device, which have led to the extensive use of I-Gel as a supraglottic airway device for anesthesia and cardiopulmonary resuscitation since 2007. Its recognized complications include laryngeal spasm, laryngeal trauma, sore throat, regurgitation of stomach contents, vomiting, neural injury, vocal cord paralysis, trauma to the tongue or sublingual nerves, and dysesthesia or cyanosis of the tongue (14-17). 


\section{Objectives}

This study was designed to compare the success rate of airway management using LMA and I-Gel in patients undergoing elective orthopedic surgeries. The insertion time, failure rate, and incidence of complications including sore throat, bleeding, and hoarseness were compared between study groups.

\section{Patients and Methods}

The study was approved by Research Ethics Committee of Iran University of Medical Sciences, registered at Iranian registry of clinical trials site (ID: IRCT201307284969N7) and written informed consent was obtained from all subjects. This single-blinded randomized clinical trial was performed on patients requiring minor orthopedic surgeries (with shorter than one hour duration) such as knee arthroscopy and malleolus fracture in Firoozgar Hospital, Iran University of medical Sciences, Tehran, Iran. Sample size was calculated at 26 in each group based on statistical significance level of $=0.05$ and $\mathrm{B}=0.90$ to detect $40 \%$ differences in success rate of insertion (18). After excluding 12 patients (due to different reasons) (Figure 1), the remained 61 patients were randomly allocated to two groups of LMA $(\mathrm{n}=31)$ and I-Gel $(\mathrm{n}=30)$ using block randomization. The inclusion criteria were age range of 18 to 70 years, complete NPO time (empty stomach), ASA Class 1 and 2, requiring minor orthopedic surgeries (estimated duration $<1$ hour), airway characteristics of thyromental distance (TMD) $>60 \mathrm{~mm}$, Mallampati score of one and two, mouth opening $>$ two finger, absence of reflux gastroesophageal reflux disease, body mass index $<30 \mathrm{~kg} /$ $\mathrm{m}^{2}$, no contraindication for the anesthetic or the muscle relaxant agents, and no history of difficult airway in previous operations. After transferring the patients to the operating table, an intravenous catheter was placed in their hand and the subjects received standard monitoring of ECG, heart rate, oxygen saturation, and blood pressure. Anesthesia was induced as follows:

First, $5 \mathrm{~mL} / \mathrm{kg}$ crystalloid IV fluids were administered and then the patients were preoxygenated for three minutes. The patients received $0.02 \mathrm{mg} / \mathrm{kg}$ midazolam and $2 \mu \mathrm{g} / \mathrm{kg}$ fentanyl for premedication. Anesthesia was induced by the administration of $5 \mathrm{mg} / \mathrm{kg}$ thiopental and $0.5 \mathrm{mg} / \mathrm{kg}$ atracurium. After three minutes of ventilation, the respective mask LMA (Haiyan kangyuan Co., China) (Figure 2) or I-Gel (Intersurgical Co., United Kingdom) (Figure 3) was placed by an anesthesiology resident (third year) who was expert in using these devices and used to insert both of them for general anesthesia for more than one year. The following LMA devices were placed for patients in LMA group:

LMA \#3 for patients weighing 30 to $50 \mathrm{~kg}$; LMA \#4 for patients weighing 50 to $70 \mathrm{~kg}$; and LMA \#5 for patients weighing 70 to $100 \mathrm{~kg}$.
The following I-Gel airways were inserted for patients in I-Gel group:

I-Gel \#3 for patients weighing 30 to $60 \mathrm{~kg}$; I-Gel \#4 for patients weighing 60 to $90 \mathrm{~kg}$; and I-Gel \#5 for patients weighing $>90 \mathrm{~kg}$.

The patients were then ventilated using a bag-valve device. Airway insertion would be considered successful if there was no air leak during ventilation and the patient had an airway pressure of $\leq 20 \mathrm{~cm} \mathrm{H}_{2} \mathrm{O}$,chest movement during ventilation, and normal pulmonary auscultation. Then the patients were ventilated by anesthesia machine (Fabius Plus, Drager, Germany) in the following settings: tidal volume, $7 \mathrm{cc} / \mathrm{kg}$; respiratory rate, $12 /$ min; and exhaled tidal volume (difference between the exhaled and inhaled volumes), $<10 \%$ (monitored by anesthesia machine). To maintain a $\mathrm{PCO}_{2}$ of 30 to $35 \mathrm{mmHg}$ on the capnograph, respiratory rate was adjusted again. The time interval between the insertions of device through the lips to the first leak-free ventilation with $20 \mathrm{~cm} \mathrm{H}_{2} \mathrm{O}$ airway pressure was considered as the insertion time and measured in seconds. If the first attempt was not successful, the device would be extracted and attempted again. No delay or ventilation would be made between insertion attempts. Up to three attempts were allowed. Number of attempts to establish adequate ventilation was recorded. If the third attempt was not successful, endotracheal tube would be used. For maintenance, $100 \mathrm{mcg} / \mathrm{kg} / \mathrm{min}$ propofol along with $\mathrm{N}_{2} \mathrm{O}$ and $\mathrm{O}_{2}$ combination (50:50) was administered. If the patient's respiration returned, $10 \mathrm{mg}$ of atracurium was re-administered. At the end of the operation, gentle circulatory oropharyngeal suctioning was performed using soft Nelaton \#12 catheter. After resumption of respiration, $0.04 \mathrm{mg} / \mathrm{kg}$ of neostigmine and $0.02 \mathrm{mg} / \mathrm{kg}$ of atropine were administered to reverse the effects of the muscle relaxant. As the patient regained adequate respiratory parameters, i.e. respiratory volume, respiratory rate, and consciousness, the device would be removed and evaluated for presence of blood on device. Moreover, the patient was evaluated for hoarseness and sore throat until discharge from the recovery room. Presence of any complication would be recorded.

Data were collected and analyzed using SPSS 11 (SPSS Inc., Chicago, IL, USA). The frequency and percentage for qualitative variables and the mean and standard deviation (SD) for quantitative variables were calculated. The quantitative data were analyzed using Student's ttest.

\section{Results}

A total of 61 subjects were evaluated including 31 patients (50.8\%) in the LMA and $30(49.2 \%)$ in the I-Gel groups. Among demographic variables, sex $(\mathrm{P}=0.001)$ (Table 1 ) and weight $(\mathrm{P}=0.006)$ (Table 2 ) were significantly different between two groups. Age was not significantly different between study groups (Table 2). Placement of LMA and I-Gel was divided into three categories regarding placement on the first, second, or third attempts. 


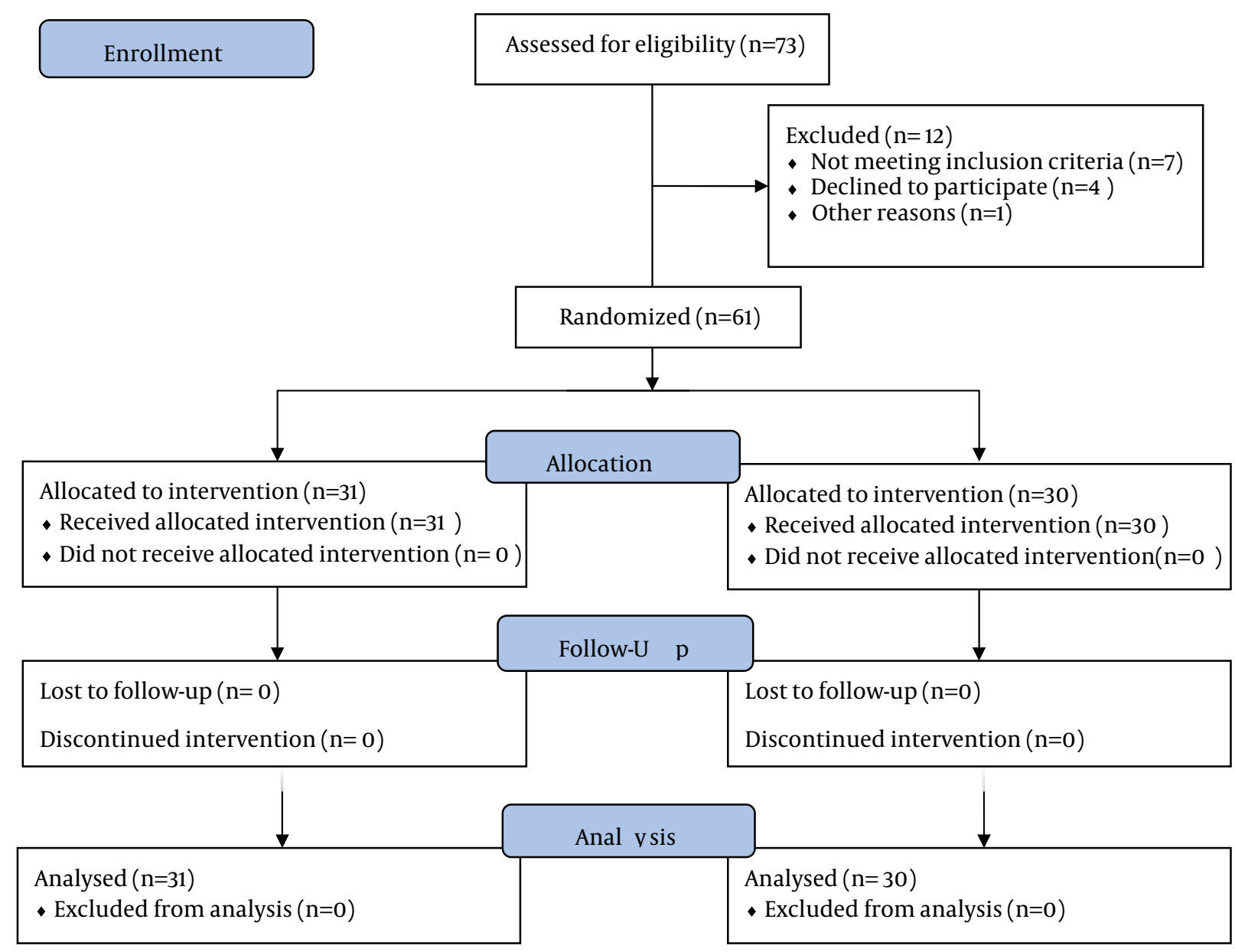

Figure 1. Flow Diagram of Patients Recruitments

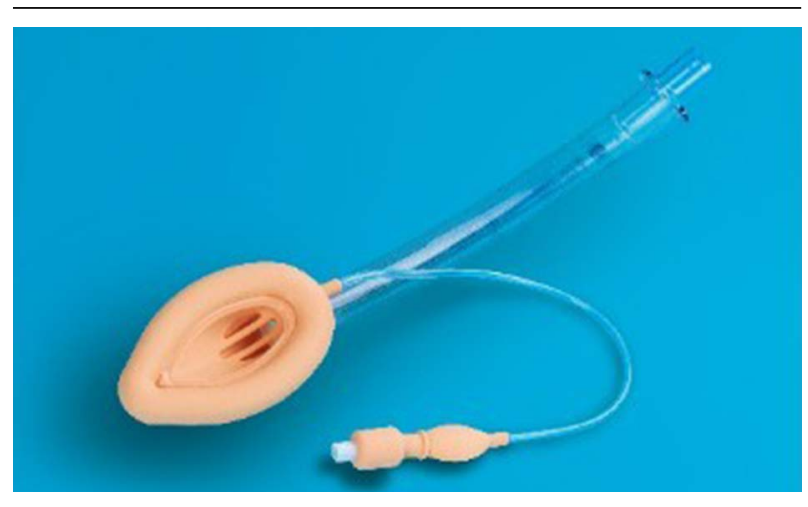

Figure 2. Laryngeal Mask Airway

Unsuccessful placement on the third attempt was considered failure and endotracheal tube was used in such cases. Results of comparison of the success rate of airway management are listed in Table 3.The difference between the two groups in regards of insertion time and

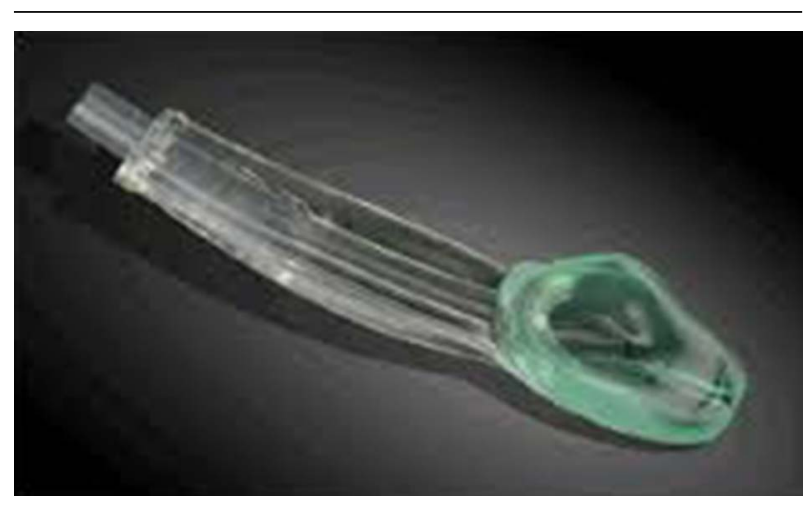

Figure 3. I-Gel

attempts were not significant. Regarding complications following airway management, bleeding was seen in none of the study groups. The frequencies of sore throat and hoarseness were not significantly different between two groups (Table 3 ). 
Pournajafian A et al.

\begin{tabular}{|c|c|c|}
\hline Gender & No. (\%) & P Value \\
\hline IGEL & & 0.001 \\
\hline Male & $7(23.3)$ & \\
\hline Female & $23(76.7)$ & \\
\hline Total & $30(100)$ & \\
\hline LMA & & 0.001 \\
\hline Male & $21(67.7)$ & \\
\hline Female & $10(32.3)$ & \\
\hline Total & $31(100)$ & \\
\hline
\end{tabular}

a Abbreviations: IGEL, supraglottic gel device; and LMA, laryngeal mask airway.

Table 2. Comparison of the Mean age and Mean Weight of Patients Between the Two Groups

\begin{tabular}{lccccc}
\hline Airway & Number & Minimum & Maximum & Mean \pm SD & P Value \\
\hline Age, $\mathbf{y}$ & & & & & 0.54 \\
I-GEL & 30 & 21 & 70 & $40.73 \pm 14.08$ & \\
LMA & 31 & 22 & 56 & $42.61 \pm 9.16$ & 0.006 \\
Weight, kg & & & & $64.10 \pm 6.98$ & \\
I-GEL & 30 & 48 & 80 & $70.41 \pm 9.89$ & \\
LMA & 31 & 45 & 87 & & \\
\hline
\end{tabular}

Table 3. Comparison of the Success Rate and Complications of Airway Management by Laryngeal Mask Airway and I-GEL

\begin{tabular}{|c|c|c|c|}
\hline Attempts, \% & LMA & IGEL & P Value \\
\hline LMA & & & 0 \\
\hline Once & 80.6 & & \\
\hline Twice & 12.9 & & \\
\hline IGEL & & & 0 \\
\hline Once & 66.7 & & \\
\hline Twice & 16.7 & & \\
\hline Thrice & 3.33 & & \\
\hline Mean Number of Attempts & 1.25 & 1.50 & 0.17 \\
\hline Insertion Time, s, Mean & 21.35 & 27.96 & 0.16 \\
\hline Hoarseness, \% & & & 0.96 \\
\hline Yes & 12.9 & 13.3 & \\
\hline No & 87.1 & 86.7 & \\
\hline Bleeding, \% & & & 0.8 \\
\hline Yes & 0 & 0 & \\
\hline No & 100 & 100 & \\
\hline Sore Throat, \% & & & 0.13 \\
\hline Yes & 19.4 & 36.7 & \\
\hline No & 80.6 & 63.3 & \\
\hline Failure Rate, \% & & & 0.36 \\
\hline Yes & 6.5 & 13.3 & \\
\hline No & 93.5 & 86.7 & \\
\hline
\end{tabular}




\section{Discussion}

According to our study, I-Gel can be inserted as fast as LMA with adequate ventilation and has no major airway complications. Therefore, it could be a good alternative to LMA in emergency airway management or general anesthesia. LMA and other supraglottic airway devices have an inflatable cuff. These cuffs can cause problems during insertion, fixation, or function of the airway. Cuff over inflation causes mechanical compression, less adaptation to the larynx, and lower airway pressure. Mechanically, cuff inflation leads to airway movement in some cases. Moreover, inflatable-cuffed laryngeal mask devices might compress the veins and cause neural injury. I-Gel is different from other supraglottic airway devices due to having an acceptable stiffness and a noninflatable cuff. To have a success rate similar to that of LMA, I-Gel can be a good alternative to LMA for airway management in emergency cases or when there is a lack of expertise or absence of a laryngoscope. This study aimed to compare the success rate of LMA and I-Gel for airway management in patients undergoing elective orthopedic surgery. Among demographic variables, sex and weight were significantly different between two groups; however, these differences had no effect in our study. The insertion time, failure rate, and incidence of complications such as sore throat, bleeding, and hoarseness were compared as well. The results revealed that in the majority of patients, one attempt sufficed for airway placement. Turan et al. studied on 90 patients with ASA 1 and 2, awaiting short surgical procedures, and found no significant difference in terms of hemodynamic disturbances or time between LMA and Cobra PLA (an uncuffed airway device resembling I-Gel); however, the success rate was lower for LMA (57\% versus $97 \%$ for PLA, $\mathrm{P}<0.05)$. The success rates reported for LMA in their study were lower than ours, which might be attributed to the use of Cobra PLA in their study (18). In a clinical trial by Singh et al. 48 patients aged 16 to 50 years with mouth opening and neck movement limitations, who were candidate for surgery, were managed by LMA or I-Gel devices. The ventilation time and success rate were compared between the two groups. Considering the easier insertion and higher success rate of I-Gel (91.7\%) in comparison to LMA (79.2\%) ( $P=0.000)$, they recommended the use of I-Gel for emergency situations and management of difficult airways (19). In a study by Siddiqui et al. 100 ASA class 1 and 2 surgical patients, aging15 to 75 years, were allocated to two groups of LMA and I-Gel and the compared in terms of easy placement of the airway and postoperative complications. They showed that the placement of both devices was easy $(\mathrm{P}=0.65)$ and both were considered to be suitable alternatives for each other (20). In a study by Francksen et al. (21) no failure occurred in the I-Gel group and only one case of failure was reported in the LMA (5\%). They reported similar efficacy of both devices, which was in agreement with our findings. In our study, the mean insertion time of devices was not significantly different between two groups $(\mathrm{P}=0.1)$. Uppal et al. (22) reported a mean insertion time of 12.2seconds (range, 9.7-14.3) for the I-Gel and 15.2seconds (range, 13.2-17.3) for the LMA, which were significantly different $(P$ $=0.007)$. They concluded that despite the significant difference in the insertion time, placement of both devices was easy and they had similar efficacy. In the study by Atef et al. (23), the mean insertion time in the I-Gel group was significantly shorter than was in LMA $(P=0.0023)$. In our study, no significant difference was seen between two groups in the incidence of bleeding $(\mathrm{P}=0.8)$, sore throat $(\mathrm{P}=0.13)$, and hoarseness $(\mathrm{P}=0.96)$. In a study by Turan et al. (18), 50\% of patients in the PLA group had sore throat, which was significantly different from the rate in LMA group $(\mathrm{P}<0.05)$. Azarsina et al. reported that patients with LMA had higher incidence of dysphagia in comparison to patients with I-Gel (24). Independent-samples $t$ test revealed a significant difference in this respect $(\mathrm{P}=$ 0.001). They reported a significant difference in the prevalence of dysphagia in compared to insignificant difference in this respect in ours, which might be attributed to the higher number of patients in their work.

Limitations of the study. Only low-risk patients (ASA class 1 and 2) with normal airways were studied, power of $40 \%$ was selected because of $35 \%$ difference in insertion success rate in previous studies. Our study reported no significant postoperative complication following the use of LMA or I-Gel. I-Gel can be used for airway management in emergency setting or when there is a lack of expertise or absence of a laryngoscope, the same as and as quick as LMA; however, its use in major surgeries or high-risk patients requires further investigations.

\section{Acknowledgements}

We wish to thank all anesthesia technicians who helped us and the patients who participated in the study.

\section{Funding/Support}

This study was supported by Firoozgar Hospital, Tehran, Iran.

\section{References}

1. James CD. Sir William Macewen and anaesthesia. Anaesthesia. 1974;29(6):743-53.

2. European Resuscitation Council (ERC), American Heart Association (AHA), International Liaison Committee on Resuscitation (ILCOR. International Guidelines 2000 for Cardiopulmonary Resuscitation and Emergency Cardiac Care. 2000;6.

3. Choyce A, Avidan MS, Harvey A, Patel C, Timberlake C, Sarang K, et al. The cardiovascular response to insertion of the intubating laryngeal mask airway. Anaesthesia. 2002;57(4):330-3.

4. Takita K, Morimoto Y, Kemmotsu O. Tracheal lidocaine attenuates the cardiovascular response to endotracheal intubation. Can J Anaesth. 2001;48(8):732-6.

5. Ziyaeifard M, Azarfarin R, Ferasatkish R, Dashti M. Management of Difficult Airway With Laryngeal Mask in a Child With Mucopolysaccharidosis and Mitral Regurgitation: A Case Report. Res Cardiovasc Med. 2014;3(2):e22068.

6. Taregh SH, Katbab MR, Katbab A, Eghbal H, Amini A. Comparison of Intraocular Pressure and Hemodynamic Response Subse- 
quent to Tracheal Tube versus Laryngeal Tube Insertion during General Anesthesia. J Res Med Sci. 2009;11(1):52-6.

7. Ziyaeifard M, Azarfarin R, Massoumi G. A comparison of intraocular pressure and hemodynamic responses to insertion of laryngeal mask airway or endotracheal tube using anesthesia with propofol and remifentanil in cataract surgery.J Res Med Sci. 2012;17(6):503-7.

8. Peppard SB, Dickens JH. Laryngeal injury following short-term intubation. Ann Otol Rhinol Laryngol. 1983;92(4 Pt 1):327-30.

9. Jayashree S. Laryngeal mask airway and its variants. Indian J Anaesth. 2005;49:275-80.

10. Weiler N, Latorre F, Eberle B, Goedecke R, Heinrichs W. Respiratory mechanics, gastric insufflation pressure, and air leakage of the laryngeal mask airway. Anesth Analg. 1997;84(5):1025-8.

11. Barker P, Langton JA, Murphy PJ, Rowbotham DJ. Regurgitation of gastric contents during general anaesthesia using the laryngeal mask airway. BrJ Anaesth. 1992;69(3):314-5.

12. Tabari M, Alipour M, Ahmadi M. Hemodynamic changes occurring with tracheal intubation by direct laryngoscopy compared with intubating laryngeal mask airway in adults: A randomized comparison trial. Egypt J Anaesth. 2013;29:103-7.

13. Levitan RM, Kinkle WC. Initial anatomic investigations of the Igel airway: a novel supraglottic airway without inflatable cuff. Anaesthesia. 2005;60(10):1022-6.

14. Lange M, Smul T, Zimmermann P, Kohlenberger R, Roewer N, Kehl F. The effectiveness and patient comfort of the novel streamlined pharynx airway liner (SLIPA) compared with the conventional laryngeal mask airway in ophthalmic surgery. Anesth Analg. 2007;104(2):431-4.

15. Michalek P, Hodgkinson P, Donaldson W. Fiberoptic intubation through an I-gel supraglottic airway in two patients with predicted difficult airway and intellectual disability. Anesth Analg.
2008;106(5):1501-4.

16. Gatward JJ, Cook TM, Seller C, Handel J, Simpson T, Vanek V, et al Evaluation of the size 4 i-gel airway in one hundred non-paralysed patients. Anaesthesia. 2008;63(10):1124-30.

17. Soar J. The I-gel supraglottic airway and resuscitation--some initial thoughts. Resuscitation. 2007;74(1):197.

18. Turan A, Kaya G, Koyuncu O, Karamanlioglu B, Pamukcu Z. Comparison of the laryngeal mask (LMA) and laryngeal tube (LT) with the new perilaryngeal airway (CobraPLA) in short surgical procedures. Eur J Anaesthesiol. 2006;23(3):234-8.

19. Singh J, Yadav MK, Marahatta SB, Shrestha BL. Randomized crossover comparison of the laryngeal mask airway classic with i-gel laryngeal mask airway in the management of difficult airway in post burn neck contracture patients. Indian J Anaesth. 2012;56(4):348-52.

20. Siddiqui AS, Raees US, Siddiqui SZ, Haider S, Raza SA. Comparison of performance and safety of I-gel with laryngeal mask airway (classic) for general anaesthesia with controlled ventilation. Anaesth Pain Intensive Care. 2010;14(1):17-20.

21. Francksen H, Renner J, Hanss R, Scholz J, Doerges V, Bein B. A comparison of the i-gel with the LMA-Unique in non-paralysed anaesthetised adult patients. Anaesthesia. 2009;64(10):1118-24.

22. Uppal V, Fletcher G, Kinsella J. Comparison of the i-gel with the cuffed tracheal tube during pressure-controlled ventilation. $\mathrm{Br}$ Anaesth. 2009;102(2):264-8.

23. Atef HM, Helmy AM, El-Taher EM, Henidak AM. Comparative study between I-gel, a new supraglottic airway device, and classical laryngeal mask airway in anesthetized spontaneously ventilated patients. Middle East J Anaesthesiol. 2012;21(4):583-90.

24. Azarsina $\mathrm{H}$. The supraglottic airway I-gel in comparison with laryngeal mask airway in anesthetized patients. Eur J Anestesiologia. 2013. 